\title{
Maximising the sensitivity of next generation multi-object spectroscopy: system budget development and design optimizations for the Maunakea Spectroscopic Explorer
}

\author{
Alan W. McConnachie ${ }^{\mathrm{a}, \mathrm{b}}$, Nicolas Flagey ${ }^{\mathrm{a}}$, Kei Szeto ${ }^{\mathrm{a}}$, Shan Mignot ${ }^{\mathrm{c}}$, Alexis Hill ${ }^{\mathrm{a}}$, and Pat \\ Hall $^{\mathrm{d}}$

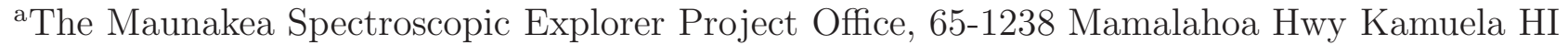 \\ 96743 USA \\ ${ }^{\mathrm{b}}$ NRC Herzberg, Dominion Astrophysical Observatory, 5071 West Saanich Road, Victoria, \\ British Columbia, Canada \\ ${ }^{\mathrm{c}}$ GEPI, Observatoire de Paris, PSL Research University, CNRS, Univ. Paris Diderot, Sorbonne \\ Paris Cité, Place Jules Janssen, 92195 Meudon, FRANCE \\ ${ }^{\mathrm{d}}$ Department of Physics and Astronomy, York University, Toronto, ON M3J 1P3, Canada
}

\begin{abstract}
MSE is an $11.25 \mathrm{~m}$ telescope with a 1.5 sq.deg. field of view. It can simultaneously obtain 3249 spectra at $R=3000$ from $360-1800 \mathrm{~nm}$, and 1083 spectra at $R=40000$ in the optical. Absolutely critical to the scientific success of MSE is to efficiently access the faint Universe. Here, we describe the adopted systems engineering methodology to ensure MSE meets the challenging sensitivity requirements, and how these requirements are partitioned across three budgets, relating to the throughput, noise and fiber injection efficiency. We then describe how the sensitivity of MSE as a system was estimated at the end of Conceptual Design Phase, and how this information was used to revisit the system design in order to meet the sensitivity requirements while maintaining the overall architectural concept of the Observatory. Finally, we present the anticipated sensitivity performance of MSE and describe the key science that these capabilities will enable.
\end{abstract}

Keywords: Manuscript format, template, SPIE Proceedings, LaTeX

\section{INTRODUCTION}

The Maunakea Spectroscopic Explorer (MSE) is the only dedicated, optical and near-infrared, large aperture $(>10 \mathrm{~m})$, multi-object spectroscopic facility being designed for first light in the mid-2020s. It is a re-purposing of the Canada-France-Hawaii Telescope, within an expanded international partnership and upgraded to a larger aperture.

A key aspect of MSE science is the spectroscopic follow-up of the plethora of astrophysical objects identified by current and future photometric and astrometric surveys at a range of wavelengths. This includes facilities like Gaia, LSST, SKA, Euclid and WFIRST. Here, there are literally billions of sources, for which exquisite multiwavelegth data will be available. However, a major missing link in this network of facilities and data is optical and near-infrared spectroscopy, and it is this gap that MSE is designed to fill.

The overwhelming majority of all sources identified by these precursor surveys are faint, and there already exists many MOS spectrographs and facilities at smaller (4m and less) aperture. MSE must therefore reach the fainter objects that cannot be accessed with smaller facilities. Overall system sensitivity is therefore critical. This paper describes the methodology and design of MSE to meet the demanding sensitivity requirements, and discusses ways in which the design is being iterated to ensure the sensitivity of MSE is unrivaled and enables new science.

Further author information: (Send correspondence to A.W.M.)

A.W.M.: E-mail: mcconnachie@mse.cfht.hawaii.edu, Telephone: 1-808-885-3188 
At the previous SPIE Astronomical Telescopes and Instrumentation meeting, the status and progress of the project were detailed in Ref. 1 while an overview of the project design was given in Ref. 2 and the science based requirements were explained in Ref. 3. An update of the project at the end of conceptual design phase is presented this year in Ref. 4 with a review of the instrumentation suite in Ref. 5. Other papers related to MSE are focusing on: the summit facility upgrade (Ref. 6,7), the telescope optical designs for MSE (Ref. 8), the telescope structure design (Ref. 9), the design for the high-resolution (Ref. 10,11) and the low/moderate-resolution spectrograph (Ref. 12, the top end assembly (Ref. 13,14), the fiber bundle system (Ref. 15,16), the fiber positioners system (Ref. 17), the systems budgets architecture and development (Ref. 18,19), the observatory software (Ref. 20), the spectral calibration (Ref. 21,22), the throughput optimization (Ref. 23, 24), the observing efficiency (Ref. 25), and the overall operations of the facility (Ref. 26).

Section 2 describes the science requirements on the system sensitivity for MSE. Section 3 describes the formalism by which sensitivity requirements are translated into high level requirements on system throughput, noise sources, and injection efficiency. Section 4 describes the allocation of values to these three child budgets, and Section 5 describes the current estimates for these quantities based on the conceptual design results. Section 6 describes the design optimisations that are being considered by the Project Office to reconcile the design with the requirements, and Section 7 discusses next steps and summarises.

\section{SCIENCE REQUIREMENTS ON SENSITIVITY}

There are three high level science requirements on sensitivity given in the science requirements document. The sensitivity requirements, like all science requirements, have been derived from consideration of the science described in the Detailed Science Case, ${ }^{27}$ and in particular the Science Reference Observations. The SROs describe, in considerable detail, specific and transformational science programs that are uniquely possible with MSE. Science requirements are defined as the core set of science capabilities that MSE must have in order to enable the science described by the SROs.

There are three science requirements relating to sensitivity, one each for the low, moderate and high resolution mode of the facility/instrument. They all have the same form and describe the signal-to-noise ratio that must be obtained for an observation of a source with a particular intrinsic flux level. The sensitivity science requirements for the low/moderate/high resolution modes are as follows:

- REQ-SRD-034/035/036: Sensitivity at [low/moderate/high] resolution

In the [low/moderate/high] resolution mode, an extracted spectrum from MSE taken in the observing conditions described below shall have a signal to noise ratio per resolution element at a given wavelength that is greater than or equal to $[2 / 2 / 10]$ for a 1 hour observation of a point source with a flux density of $[0.91 / 1.4 / 36] \times 10^{-29} \mathrm{ergs} / \mathrm{sec} / \mathrm{cm}^{2} / \mathrm{Hz}$ at that wavelength, for all wavelengths longer than $400 \mathrm{~nm}$. Between $370-400 \mathrm{~nm}$, the SNR shall not be less than $[1 / 1 / 5]$ at any wavelength. The observing conditions in which this requirement shall be met correspond to a sky brightness of $[20.7 / 20.7 / 19.5]$ mags/sq.arcsec in the Vband at an airmass of 1.2 , and a delivered image quality at that airmass of 0.6 arcseconds full width at half maximum in the $\mathrm{r}$ band.

MSE is expected to undertake observations of many different sources with different luminosities, exposure times and observing conditions. Thus, the characteristics of the observation described in the science requirement should be considered a "reference" observation only, whose sole purpose is to define the sensitivity of the MSE system.

\section{METHODOLOGY}

\subsection{Overview}

In order to meet the science requirements for sensitivity, it is necessary to translate the requirement on SNR (sensitivity) as a function of wavelength for each mode of MSE into requirements (budgets) on the principal factors that contribute to the SNR calculation. In Section 3.2, we show that these principal factors are the 
system throughput, the fiber injection efficiency (IE, the fraction of light from a point source that is incident on the focal plane that enters a science fiber) and the noise (specifically, the random sources of noise that occur during an observation; systematics errors are not considered part of the noise budget). The formalism developed in Section 3.2 also describes the inter-relation of each of these components with each other (such that if one changes, the others must change accordingly to ensure the SNR requirement is still met).

In what follows, we make an intentional distinction between budget/allocation and estimate. The former is the top-down requirement which is driven by the science considerations. The latter is a bottom-up value, which is considered feasible given our current knowledge of the subsystem conceptual designs for MSE. If the latter is very different from the former (such that the estimate does not meet the allocation), then this implies a potential risk for the project as it highlights an area where we might fail to meet the requirements of the Project. Clearly, it is the responsibility of the Project Office to reconcile any discrepancies between them in order to achieve the MSE science goals. If meeting these science goals ultimately prove infeasible, then a revist of the science requirements, and thus on the scientific capabilities of MSE, might be required.

Thus, the process the Project Office has followed to maximise the sensitivity of MSE, and which is described in detail in the proceeding sections, is as follows:

1. translate the science requirements on sensitivity into high-level budgets for injection efficiency, system throughput and noise (following Section 3.2);

2. partition each of these three child budgets into budget allocations for each of the relevant subsystems;

3. compare the performance estimates from the conceptual designs of all the subsystems to the budget allocations;

4. revisit the budget allocations and/or designs to reconcile the allocations and the estimates where necessary;

5. in cases where reconciliation is possible but difficult, a risk will be identified by the Project Office that will need to be managed;

6. in cases where reconciliation is not possible, the high level science requirements may need to be modified. This is a serious step to take that requires ultimate approval by the MSE Management Group.

\subsection{SNR Formalism}

For an astronomical observation, the SNR is defined as

$$
S N R=N_{o b j} / \sqrt{N_{\text {noise }}}
$$

where

$$
N_{\text {noise }}=N_{o b j}+N_{\text {sky }}+N_{\text {other }}^{2}
$$

Here, $N_{o b j}$ is the number of counts per resolution element due to the astronomical object being observed, $N_{s k y}$ is the number of counts per resolution element due to the sky background, and $N_{\text {other }}$ are the counts (or effective counts) due to all other sources of noise (for example, detector read noise and dark current).

For an astronomical source with an intrinsic flux of $F_{o b j}$, observed for a time $t$, by a telescope with a collecting aperture of area $S$, operating a fiber spectrograph with a wavelength resolution of $\Delta \lambda$ :

$$
N_{o b j}=\left(F_{o b j} \times \Delta \lambda \times t \times S \times E\right) / P
$$

$P=h c / \lambda$ is the energy per photon, where $h$ is Plank's constant and $c$ is the speed of light. $E$ is the overall efficiency (throughput) of the entire astronomical system including atmosphere. We choose to deconstruct $E$ as follows: 


$$
E=E_{a t m} \times E_{i n j} \times E_{s}
$$

$E_{a t m}$ is the throughput of the atmosphere. The product of $\left(E_{i n j} \times E_{s}\right)$ is the overall throughput of the telescope and instrument (from primary mirror to detectors). That is, it describes how much of the flux from the astronomical target that enters the telescope (is incident on M1) is measured by the instrument detectors.

Excluding the atmosphere, we have chosen to break the throughput into two components. The first component is the injection efficiency, $E_{i n j}$, which is the fraction of light from the astronomical source incident on the focal plane of the telescope that makes it into the fiber. The second term, $E_{s}$, refers to the combined throughput of all other components of the telescope and instrument.

The reason that we have chosen to define the throughput in this way is clear when we consider the number of sky photons detected:

$$
N_{s k y}=\left(F_{s k y} \times \frac{\pi d^{2}}{4} \times \Delta \lambda \times t \times S \times E_{s}\right) / P
$$

Here, $d$ is the diameter of the fiber. Adopting these definitions, $E_{s}$ can be used in the definition of both the object counts and the sky counts.

Substituting everything into Equation (2), and making explicit the dependency on wavelength, gives the following:

$$
\frac{S N R(\lambda)=\left(\left(F_{o b j}(\lambda) \times \Delta \lambda \times t \times S \times E_{a t m}(\lambda) \times E_{i n j}(\lambda) \times E_{s}(\lambda)\right) / P(\lambda)\right) /}{\sqrt{\left(F_{o b j}(\lambda) \times \Delta \lambda \times t \times S \times E_{a t m}(\lambda) \times E_{i n j}(\lambda) \times E_{s}(\lambda)\right) / P(\lambda)+\left(F_{s k y}(\lambda) \times \frac{\pi d^{2}}{4} \times \Delta \lambda \times t \times S \times E_{s}(\lambda)\right) / P+N_{o t h e r}(\lambda)^{2}}}
$$

All relevant terms in Equation (6), except $E_{s}, E_{i n j}$ and $N_{\text {other }}$ are defined in the science requirements of MSE or through the design choices of the Observatory described in the MSE Observatory Architecture Document. The values of all known parameters in Equation 6 are summarised in Table 1. We note the following:

- The object flux levels correspond to sources with monochromatic magnitudes of $m=24.0 / 23.5 / 20$ for the low resolution, moderate resolution and high resolution modes, respectively;

- We use the ESO SkyCalc ${ }^{28,29}$ model values, modified for Maunakea, for the sky emission spectrum and the atmospheric absorption. We use a highly smoothed version of the simulated sky spectrum to avoid the influence of the many strong emission lines, as shown by the red line in Figure 1.

Thus, to meet the overall sensitivity requirements of MSE requires partitioning appropriate values to the system throughput, injection efficiency and noise terms of Equation (6) given the adopted values of all the other parameters described in Table 1.

\section{DEVELOPMENT OF SENSITIVITY BUDGETS}

The formalism developed in the previous section allows us trade performance in the areas of system throughput, noise and IE against each other to meet the overall sensitivity requirements. As the design matures and our knowledge of the system performance improves, this three-way trade will be a critical tool at the disposal of the Project Office to ensure MSE meets its demanding sensitivity requirements.

Prior to having all information available on all relevant aspects of the MSE performance, we choose to construct the first versions of the three child budgets (IE, throughput and noise) in the following way:

First, we estimate the expected IE by conducting detailed modeling based on existing design information as well as engineering experience. We directly adopt these estimates as the allocation to the IE budget. Our 
Table 1. Summary of all relevant terms for necessary for the calculation of the SNR, as described by Equation (6)

\begin{tabular}{|c|c|c|}
\hline Parameter & Value & Notes \\
\hline$S N R(\lambda)$, Signal-to-noise ratio & $\begin{array}{l}\mathrm{LR}: \leq 400 \mathrm{~nm}-1 />400 \mathrm{~nm}-2 \\
\mathrm{MR}: \leq 400 \mathrm{~nm}-1 />400 \mathrm{~nm}-2 \\
\mathrm{HR}: \leq 400 \mathrm{~nm}-5 />400 \mathrm{~nm}-10\end{array}$ & Reference values only \\
\hline$F_{o b j}(\lambda)$, Flux from object & $\begin{array}{l}\text { LR: } 0.91 \times 10^{-29} \mathrm{ergs} / \mathrm{sec} / \mathrm{cm}^{2} / \mathrm{Hz} \\
\text { MR: } 1.4 \times 10^{-29} \mathrm{ergs} / \mathrm{sec} / \mathrm{cm}^{2} / \mathrm{Hz} \\
\text { HR: } 36 \times 10^{-29} \mathrm{ergs} / \mathrm{sec} / \mathrm{cm}^{2} / \mathrm{Hz}\end{array}$ & Reference values only \\
\hline$F_{s k y}(\lambda)$, Flux from sky & $\begin{array}{l}\text { ESO SkyCalc model normalised to: } \\
\text { LR: } V=20.7 \mathrm{mags} / \mathrm{sq} \cdot \operatorname{arcsec} \\
\text { MR: V }=20.7 \mathrm{mags} / \mathrm{sq} \cdot \operatorname{arcsec} \\
\text { HR: } V=19.5 \mathrm{mags} / \mathrm{sq} \cdot \operatorname{arcsec}\end{array}$ & $\begin{array}{l}\text { Modified for Maunakea; Reference value } \\
\text { only }\end{array}$ \\
\hline$E_{a t m}(\lambda)$, atmospheric extinction & ESO SkyCalc model, airmass 1.2 & Modified for Maunakea \\
\hline$t$, exposure time (reference) & $3600 \mathrm{~s}$ & Reference value only \\
\hline$S$, collecting area of M1 & $80.8 \mathrm{~m}^{2}$ & Design choice \\
\hline $\begin{array}{l}\Delta \lambda=\frac{\lambda}{R}, \text { wavelength resolution of } \\
\text { spectrograph }\end{array}$ & $\begin{array}{l}\text { LR: } R=3000 \\
\text { MR: } R=6000 \\
\text { HR: } \leq 500 \mathrm{~nm}-\mathrm{R}=40000 \\
\mathrm{HR}:>500 \mathrm{~nm}-\mathrm{R}=20000\end{array}$ & Set by science requirements on resolution \\
\hline$d$, diameter of fiber & $\begin{array}{l}\text { LR: } 1.0 \text { arcsecs } \\
\text { MR: } 1.0 \operatorname{arcsecs} \\
\text { HR: } 0.8 \text { arcsec }\end{array}$ & $\begin{array}{l}\text { Design choice based on optimal values } \\
\text { given expected image quality }\end{array}$ \\
\hline$P(\lambda)$, energy of photon & $\frac{h c}{\lambda}$ & Fundamental physics \\
\hline
\end{tabular}

rationale for doing this is that we expect that there is limited design alternatives available that can change the IE significantly, given that we are already pushing extremely hard on the IE to make it a maximum.

We then estimate all the known sources of subsystem noise, based on demonstrated performance of components, existing design information, and engineering experience. Some of these sources of noise depend upon the final signal (e.g., the expected diffuse light level is a fraction of the average signal), and so we note that their absolute values depend upon the overall throughput (including IE), which is initially unknown. These values (either absolute noise levels or noise levels relative to the signal) are then allocated to the noise budget.

Finally, we use a custom code to solve the set of equations that we describe in Section 3 to determine what the throughput budget allocation must be in order to meet the SNR requirements described in the SRD. This involves an iterative process whereby the absolute values of the noise budgets are revised each time the throughput is calculated, and this process is repeated until convergence.

As the previous section makes clear, the three child budgets of sensitivity are not independent, and so at the very least some elements of each of the budgets would have to be calculated - in contrast to being directly allocated - in order to ensure consistency with the driving science requirements. In this first version of these budgets, we have chosen to calculate, rather than independently allocate, the system throughput. Our rationale for this is that we expect there to be a bigger design-space to explore to find suitable solutions that can increase system throughput than there is to increase injection efficiency and to minimise most sources of noise compared to our baseline assumptions on these quantities (see next section). Even if this rationale is wrong, the formalism outlined in the previous section provides a clear way for us to rebalance the budgets once we have new information on the expected performance of the various subsystems of MSE.

Table 2 presents the initial allocations to the injection efficiency, noise and system throughput budgets calculated in the way described above.

\section{ESTIMATION OF REALIZED PERFORMANCE}

The major system elements that are identified of importance for the sensitivity of MSE are the following (considering successive "Product Breakdown Structure" (PBS) elements along the optical path):

1. MSE.ENCL: the enclosure

2. MSE.TEL.STR: the telescope structure 


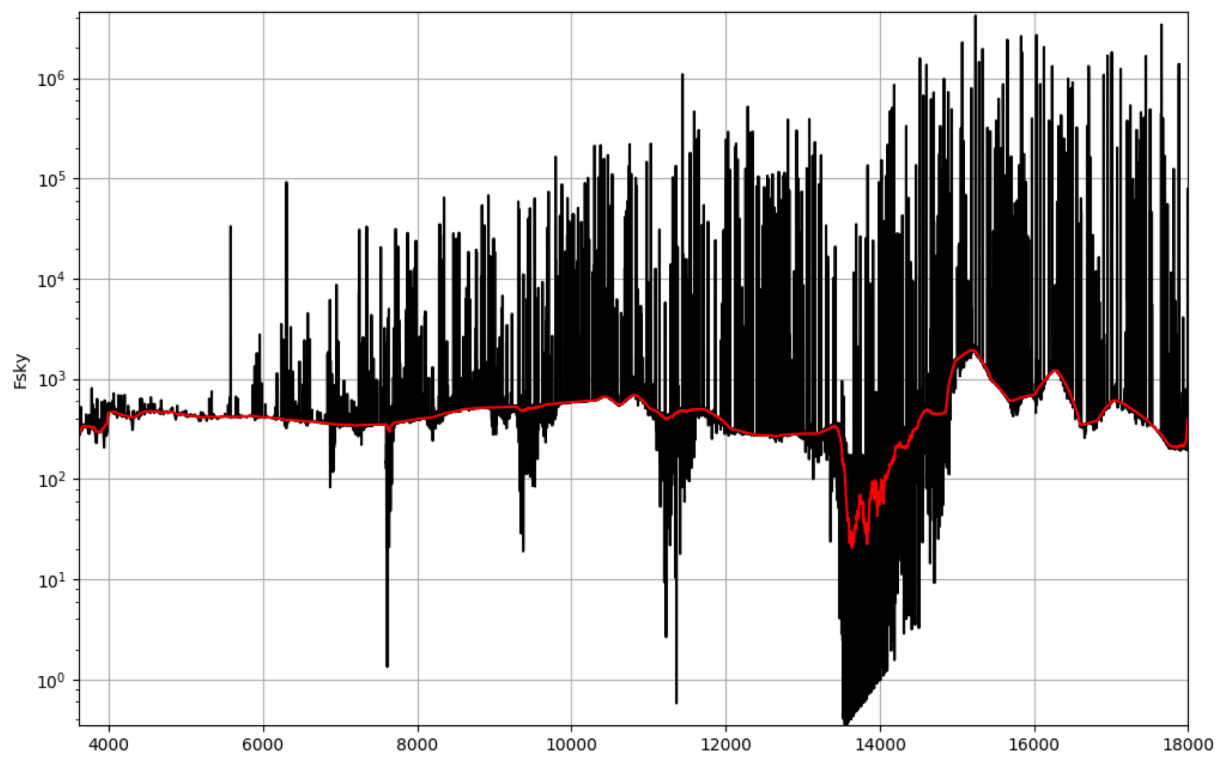

Figure 1. Example of the sky spectrum used in sensitivity (SNR) related calculations. Specifically, we use the medianfiltered version of the spectrum shown as the red line that traces the continuum level, and which does not include the many strong emission lines.

Table 2. Initial budget allocations to Injection Efficiency (\% throughput), Noise (electrons per resolution element) and System Throughput (\%).

\begin{tabular}{|c|c|c|c|c|c|c|c|c|c|c|c|c|c|c|c|c|}
\hline & $\lambda, \mathrm{nm}$ & 360 & 370 & 400 & 482 & 626 & 767 & 900 & 910 & 950 & 962 & 1235 & 1300 & 1500 & 1662 & 1800 \\
\hline Injection & LR & 66.4 & 67.8 & 70.4 & 73.0 & 74.9 & 75.6 & 76.0 & 76.0 & 76.1 & 76.1 & 76.1 & 75.8 & 74.8 & 73.2 & 71.9 \\
\hline efficiency & MR & 66.4 & 67.8 & 70.4 & 73.0 & 74.9 & 75.6 & 76.0 & 76.0 & 76.1 & & & & & & \\
\hline$(\%)$ & $\mathrm{HR}$ & 51.8 & 53.3 & 56.0 & 58.7 & 61.1 & 62.1 & 62.6 & & & & & & & & \\
\hline Noise & LR & 15 & 15 & 15 & 17 & 17 & 17 & 20 & 20 & 20 & 88 & 88 & 88 & 105 & 89 & 93 \\
\hline$\left(e^{-} /\right.$res. & MR & 14 & 14 & 15 & 15 & 15 & 15 & 17 & 17 & 17 & & & & & & \\
\hline element) & $\mathrm{HR}$ & 17 & 17 & 17 & 18 & 18 & 18 & 18 & & & & & & & & \\
\hline System & LR & 5 & 4 & 4 & 12 & 10 & 10 & 15 & 15 & 17 & 29 & 24 & 24 & 81 & 30 & 82 \\
\hline Thruput & MR & 5 & 5 & 5 & 11 & 10 & 10 & 14 & 14 & 16 & & & & & & \\
\hline (\%) & $\mathrm{HR}$ & 10 & 10 & 9 & 21 & 8 & 7 & 7 & & & & & & & & \\
\hline
\end{tabular}


3. MSE.TEL.M1: the primary mirror

4. MSE.TEL.PFHS: the prime focus hexapod system

5. MSE.TEL.WFC/ADC: the wide field corrector and atmospheric dispersion corrector

6. MSE.TEL.InRo: the instrument rotator

7. MSE.SIP.PosS: the fibre positioner system

8. MSE.SIP.FiTS: the fibre transmission system

9. MSE.SIP.LMR or MSE.SIP.HR: the spectrographs, either low/moderate (these two modes are realised in a single spectrograph system) or high resolution

All PBS elements up to and including the instrument rotator are in common for all three resolution modes. For MSE.SIP.PosS, the low and moderate resolution modes share the same fibers/positioners, different to those of the high resolution mode. For the remaining elements of the MSE.SIP branch (the "Science Instrument Package"), the subsystems are different for each resolution mode of MSE. Specifically, two different fiber systems lead to two different spectrograph locations. $\mathrm{A} \sim 30 \mathrm{~m}$ fiber cable leads to the telescope platforms, and feeds into the low/moderate spectrographs. A seperate $\sim 50 \mathrm{~m}$ fiber cable leads to the coude room in the telescope pier. This stable environment houses the high resolution spectrograph suite.

Each of these PBS elements have associated throughputs (e.g., vignetting effects, reflectivities, transmissions, etc), and contribute to the overall system throughput.

Contributions to the noise are made in the spectrographs, especially the detector system but elsewhere too (e.g., read noise, dark current, thermal background, cross-talk, ghosting, instrumental stray light). Telescope stray light is also a consideration, and directly affects TEL.STR. Point spread function considerations also impact the noise estimates (especially via cross-talk).

The IE budget is discussed in detail in Ref. 30. A critical budget that is related to the IE budget is the Image Quality budget, that describes the expected contributions to the IQ as measured at the focal plane of MSE: bigger images due to poorer IQ mean that less light is able to enter the fiber. In the first version of these budgets, we assumed that the delivered IQ was 0.6 arcsecs in the r-band, as per the sensitivity science requirement. However, subsequent work has shown that the median IQ we can expect is significantly better than this, and we discuss the impact of this improvement in the next section.

In 2017, conceptual design studies were completed for all the major subsystems (10 different reviews for 8 different subsystems). As part of these studies, throughputs for all major subsystems were estimated based on actual design information, and a much more detailed understanding of the system - include noise effects - was able to be provided than was available in earlier phases. These estimates were combined via Equation 6 to determine an estimate for the sensitivity of MSE, to compare to the science requirement described in Section 2.

Figure 2 shows the estimates (red lines) of the sensitivity of MSE as a function of wavelength compared to the requirements (blue lines) for the low, moderate and high resolution modes of MSE (top, middle and bottom panels, respectively). The key points to note here are that:

- Low and moderate resolution meet science requirements in the optical except in the $4000-4500 \mathrm{~A}$ region

- NIR performance in the low resolution mode is below requirements, significantly so for the H-band;

- The high resolution performance in the blue $(<5000 \mathrm{~A})$ does not meet requirements.

\section{DESIGN OPTIMIZATION}

We now discuss the effect of changes made in either the analysis procedure, or proposed changes in the engineering design of MSE, that can improve the sensitivity performance of MSE. We note that in each following subsection, the cumulative effect of all the recommendations in the preceding subsections are considered. 

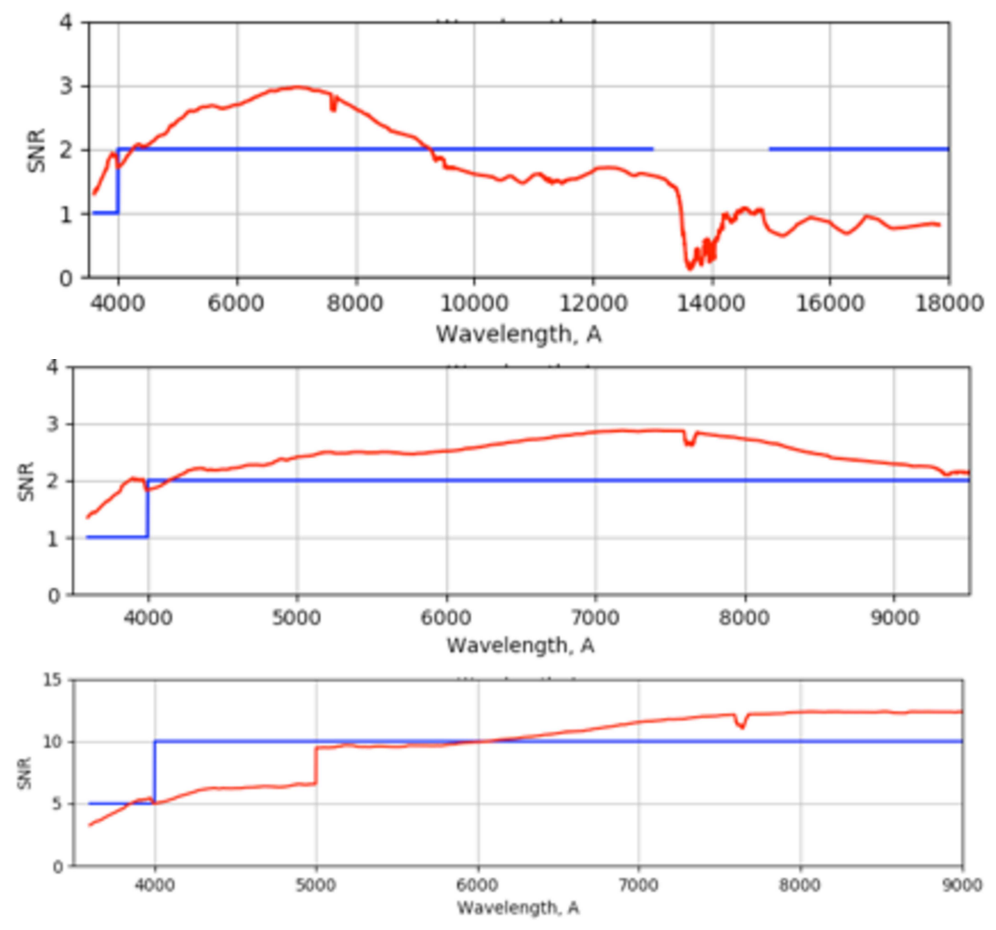

Figure 2. Initial estimates at the end of Conceptual Design for the sensitivity of the low (top panel), moderate (middle panel) and high (bottom panel) resolution modes of MSE. Blue is the requirement, red is the estimated performance.

\subsection{M1: Zecoat}

The relatively poor performance in the blue, especially for the HR mode, motivates examination of adopting a blue-enhanced coating for M1. Here, we consider the Zecoat coating as a potential candidate. The Zecoat coating improves performance significantly in the blue. It ensures we will safely meet science requirements in the low and moderate resolution modes around 4000A, and has essentially no affect at longer (> 5000A) wavelengths. Scientifically, the use of a Zecoat-like coating for M1 seems to be highly desireable.

\subsection{LMR detector operating temperature}

For the low resolution mode at NIR wavelengths, the major source of subsystem noise (as opposed to sky or object noise) is dark current. Significantly reducing the dark current will increase the sensitivity of MSE in the NIR. Our initial estimate of the sensitivity assumed a dark current of 0.05e/s/pix (H2RG@153K). However, the dark current can be significantly lower if the detector operates at a cooler temperature. Indeed, we estimate that an operating temperature of $95 \mathrm{~K}$ will give a dark current of $0.02 \mathrm{e} / \mathrm{s} / \mathrm{pix}$, more than half of what we originally adopted. An operating temperature of $75 \mathrm{~K}$ is even more desireable, since here we can anticipate a dark current of only $0.005 \mathrm{e} / \mathrm{s} /$ pix or lower. By adopting a dark current of $0.02 \mathrm{e} / \mathrm{s} / \mathrm{pix}$, we now expect to meet the low resolution science requirement in the $\mathrm{J}$ band.

\subsection{FiTS/Spectrograph locations}

Given the preciousness of blue photons in the HR mode, we are considering changing the locations of the LMR and HR, so that the HR mode can use shorter fibers (which are a major light loss especially at $<5000 \mathrm{~A}$ ). This comes at the expense of blue photons in the low and moderate resolution modes. However, it is a science requirement for the HR mode that two out of the three spectral windows that it will access are at $\lambda<5000 \mathrm{~A}$. This is due to the rich number of spectral diagnostics and chemical species that have features at blue wavelengths. In this respect, a majority of the "science content" of the HR mode is at short wavelengths, more so than in the low resolution mode. 
Our estimate on the throughput changes resulting from this switch in spectrograph locations is such that we still expect to meet the sensitivity requirement in the optical for low and moderate resolution at the blue end because of the previous changes we have incorporated (especially, the M1 ZeCoat). However, there is a notable increase in the throughput of HR at the bluest wavelengths (at $3700 \mathrm{~A}$, there is a $27 \%$ gain in throughput; at $4000 \mathrm{~A}$, there is a $13 \%$ gain). There is essentially no change at red wavelengths.

\subsection{Fiber injection}

For a given IQ, there is a theoretical best IE that can be achieved. The delivered IE is estimated from the IE budget, and we would like it to be as close to the theoretical best as possible. Generally, our modeling of the IE suggests we are around $90 \%$ of the theoretical best.

Our inital examination of this issue assumed a delivered IQ of 0.6 arcsecs in the r-band, as per the science requirements in Section 2. However, results at the end of conceptual design demonstrate that we can expect a median IQ closer to 0.5 arcsecs, not 0.6 arcsecs. With this dramatically smaller IQ, the IE for a given fiber size increases significantly. Specifically, requiring the $I Q=0.5$ instead of 0.6 essentially increases the injection efficiency by $20-25 \%$ at all wavelengths.

Clearly, pushing further on the IQ is hard. The items that contribute to it are less well understood and the uncertainty at the end of conceptual design is larger than for other budgets. However, the gain in improving the IQ by 0.1 arcseconds is immense.

\subsection{Updated sensitivity estimates}
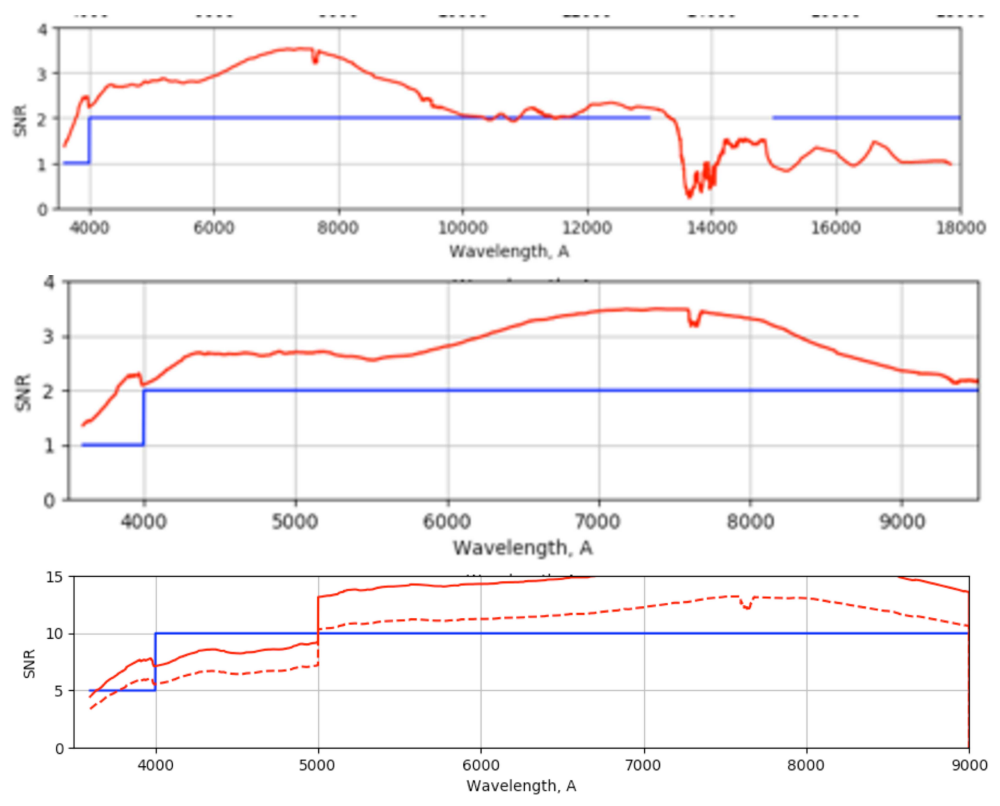

Figure 3. Updated estimates for the sensitivity of the low (top panel), moderate (middle panel) and high (bottom panel) resolution modes of MSE, after adopting the optimisations discussed in the text. Blue is the requirement, red is the estimated performance. For the high resolution mode, the solid line corresponds to the SNR level measured at the peak of the grating efficiency $(\sim 80 \%)$, and the dashed line corresponds to the SNR level measured at the minimum grating efficiency ( 50\%).

Figure 3 shows the updated estimates of the sensitivity performance of MSE after adopting the optimisations discussed previously (M1 coating, LMR operating temperature, spectrograph locations and fiber injections. We now meet the science requirements everywhere in the low and moderate resolutions except for the H-band. For the high resolution mode, two estimates are presented, corresponding to the peak throughput of the grating (solid line) and the minimum throughput (dashed line). Again, we meet science requirements everywhere except in the critical $4000-5000 \mathrm{~A}$ range. Here, however, the performance is much superior to our earlier estimates. 


\section{CONCLUSIONS AND FUTURE WORK}

MSE is on-track to be an exceptionally sensitive facility that can probe the spectra of the faintest objects in the sky. Even in the areas in which it currently formally fails to meet requirements, its estimated performance is more sensitive than any other existing large aperture multi-object spectroscopic system. For the NIR, we are currently investigating operating the H-band at lower temperatures, and also revisiting our models of the NIR sky; the latter is critical to understand correctly since sky photons are a major source of noise that limits our performance. At high resolution, new versions of the spectrograph design are being considered to increase the throughput of this challenging subsystem, including adopting off-axis collimators and reducing the fiber size, which then eases some of the stresses on the grating.

At the end of the Conceptual Design Phase, we estimate that MSE is very close to meeting the very demanding sensitivity requirements, and we are currently investigating design optimisations to reconcile those areas where the estimated performance does not meet the requirements. The proposed changes to the baseline design of MSE outlined in this paper are being discussed in the Project Office and we expect many of these recommendations will be adopted following full trade studies considering other aspects of system design not described here (cost, risk, etc).

\section{ACKNOWLEDGMENTS REFERENCES}

[1] Murowinski, R., McConnachie, A. W., Simons, D., and Szeto, K., "Maunakea Spectroscopic Explorer: the status and progress of a major site redevelopment project," in [Ground-based and Airborne Telescopes VI], Proc. SPIE 9906 (July 2016).

[2] Szeto, K., Bai, H., Bauman, S., Crampton, D., Flagey, N., Fouque, P., Gillingham, P., Gong, X., Ho, K., McConnachie, A., Mignot, S., Murowinski, R., Salmon, D., Spano, P., Zhai, C., and Zhang, K., "Maunakea Spectroscopic Explorer design development from feasibility concept to baseline design," in [Ground-based and Airborne Telescopes VI], Proc. SPIE 9906 (July 2016).

[3] McConnachie, A. W., "Science-based requirement and operations development for the Maunakea Spectroscopic Explorer," in [Ground-based and Airborne Telescopes VI], Proc. SPIE 9906 (July 2016).

[4] Szeto, K., Murowinski, R., McConnachie, A., Hill, A., Flagey, N., and Mignot, S., "Maunakea spectroscopic explorer emerging from conceptual design," in [Ground-based and Airborne Telescopes VII], Proc. SPIE 10700 (Aug. 2018).

[5] Szeto, K., Hill, A., Flagey, N., McConnachie, A., and Murowinski, R., "Maunakea spectroscopic explorer instrumentation suite," in [Ground-based and Airborne Instrumentation for Astronomy VII], Proc. SPIE 10702 (Aug. 2018).

[6] Bauman, S. E., Green, G., and Szeto, K., "Maunakea Spectroscopic Explorer observatory upgrade: a revised and optimized astronomical facility," in [Ground-based and Airborne Telescopes VI], Proc. SPIE 9906 (July 2016).

[7] Bauman, S., Szeto, K., Hill, A., Murowinski, R., Look, I., Green, G., Elizares, C., Salmon, D., Grigel, E., Manuel, E., Ruan, F., and Teran, J., "Transforming the Canada France Hawaii telescope (CFHT) into the Maunakea spectroscopic explorer (MSE): a conceptual observatory building and facilities design," in [Observatory Operations: Strategies, Processes, and Systems VII], Proc. SPIE 10704 (Aug. 2018).

[8] Saunders, W. and Gillingham, P. R., "Optical designs for the Maunakea Spectroscopic Explorer telescope," in [Ground-based and Airborne Telescopes VI], Proc. SPIE 9906 (July 2016).

[9] Murga, G., Szeto, K., Urrutia, R., Bauman, S., Bilbao, A., Lorentz, T., and Murowinski, R., "The Maunakea Spectroscopic Explorer (MSE) telescope mount," in [Ground-based and Airborne Telescopes VII], Proc. SPIE 10700 (Aug. 2018).

[10] Zhang, K., Zhu, Y., and Hu, Z., "Maunakea Spectroscopic Explorer: conceptual design of multiobject high resolution spectrograph," in [Ground-based and Airborne Instrumentation for Astronomy VI], Proc. SPIE 9908 (July 2016). 
[11] Zhang, K., Zhou, Y., Tang, Z., Saunders, W., Venn, K., Shi, J., McConnachie, A., Szeto, K., Zhu, Y., and $\mathrm{Hu}, \mathrm{C} .$, "Maunakea spectrographic explorer (MSE): preliminary design of multiobject high resolution spectrograph," in [Ground-based and Airborne Instrumentation for Astronomy VII], Proc. SPIE 10702 (Aug. 2018).

[12] Caillier, P., Saunders, W., Carton, P., Laurent, F., Migniau, J., Pcontal-Rousset, A., Richard, J., and Yche, C., "Maunakea spectroscopic explorer low moderate resolution spectrograph conceptual design," in [Ground-based and Airborne Instrumentation for Astronomy VII], Proc. SPIE 10702 (Aug. 2018).

[13] Mignot, S., Hill, A., Blin, A., Geyskens, N., and Horville, D., "Opto-mechanical design of the top end assembly (TEA) for the Maunakea spectroscopic explorer (MSE): a multi-function compact prime focus environment," in [Ground-based and Airborne Instrumentation for Astronomy VII], Proc. SPIE 10702 (Aug. 2018).

[14] Hill, A., Szeto, K., Mignot, S., and Horville, D., "Maunakea spectroscopic explorer (MSE): the prime focus subsystems: requirements and interfaces," in [Modeling, Systems Engineering, and Project Management for Astronomy VIII], Proc. SPIE 10705 (Aug. 2018).

[15] Venn, K., Monty, S., Bradley, C., Crampton, D., Erickson, D., Kielty, C., Jahandar, F., Pawluczyk, R., Fournier, P., Szeto, K., and Hill, A., "Fiber testing facility for MSE-like fiber optics," in [Ground-based and Airborne Instrumentation for Astronomy VII], Proc. SPIE 10702 (Aug. 2018).

[16] Erickson, D., Crampton, D., Pawluczyk, R., Venn, K., Hall, P., Bradley, C., McConnachie, A., Pazder, J., Jahandar, F., Kielty, C., Monty, S., Szeto, K., and Hill, A., "MSE FiTS: the ultimate multi fiber optic transmission system," in [Ground-based and Airborne Instrumentation for Astronomy VII], Proc. SPIE 10702 (Aug. 2018).

[17] Szeto, S., Baker, G., Brown, R., Gilbert, J., Gillingham, P., Saunders, W., Sheinis, A., Venkatesan, S., and Waller, L., "Sphinx: a massively multiplexed fiber positioner for MSE," in [Ground-based and Airborne Instrumentation for Astronomy VII], Proc. SPIE 10702 (Aug. 2018).

[18] Mignot, S. B., Flagey, N., Szeto, K., Murowinski, R., and McConnachie, A. W., "Systems budgets architecture and development for the Maunakea Spectroscopic Explorer," in [Modeling, Systems Engineering, and Project Management for Astronomy VI], Proc. SPIE 9911 (July 2016).

[19] Hill, A., Mignot, S., Szeto, K., Flagey, N., Murowinski, R., McConnachie, A., Hall, P., and Saunders, W., "Maunakea spectroscopic explorer (MSE): implementing the system engineering methodology for the development of a new facility," in [Modeling, Systems Engineering, and Project Management for Astronomy VIII], Proc. SPIE 10705 (Aug. 2018).

[20] Vermeulen, T. A., Isani, S., Withington, K. K., Ho, K. K. Y., Szeto, K., and Murowinski, R., "Observatory software for the Maunakea Spectroscopic Explorer," in [Software and Cyberinfrastructure for Astronomy IV], Proc. SPIE 9913 (July 2016).

[21] et al., N. F., "Spectral calibration for the maunakea spectroscopic explorer: challenges and solutions," in [Ground-based and Airborne Instrumentation for Astronomy VI], Proc. SPIE 9908 (July 2016).

[22] McConnachie, A., Hall, P., Saunders, W., and Flagey, N., "The science calibration challenges of next generation highly multiplexed optical spectroscopy: the case of the Maunakea spectroscopic explorer," in [Observatory Operations: Strategies, Processes, and Systems VII], Proc. SPIE 10704 (Aug. 2018).

[23] Flagey, N., Mignot, S. B., Szeto, K., McConnachie, A. W., and Murowinski, R., "The Maunakea Spectroscopic Explorer: throughput optimization," in [Ground-based and Airborne Instrumentation for Astronomy VI], Proc. SPIE 9908 (July 2016).

[24] McConnachie, A., Szeto, K., Hill, A., Flagey, N., Mignot, S., and Saunders, W., "Maximising the sensitivity of next generation multi-object spectroscopy: system budget development and design optimizations for the Maunakea spectroscopic explorer," in [Modeling, Systems Engineering, and Project Management for Astronomy VIII], Proc. SPIE 10705 (Aug. 2018).

[25] Flagey, N., McConnachie, A., Szeto, K., and Mahoney, B., "Expected observing efficiency of the Maunakea spectroscopic explorer," in [Observatory Operations: Strategies, Processes, and Systems VII], Proc. SPIE 10704 (Aug. 2018). 
[26] Flagey, N., McConnachie, A., Szeto, K., Hill, A., Hall, P., and Mignot, S., "Optimal scheduling and science delivery of millions of targets in thousands of fields: the operational concept of the Maunakea spectroscopic explorer (MSE)," in [Observatory Operations: Strategies, Processes, and Systems VII], Proc. SPIE 10704 (Aug. 2018).

[27] McConnachie, A., Babusiaux, C., Balogh, M., Driver, S., Côté, P., Courtois, H., Davies, L., Ferrarese, L., Gallagher, S., Ibata, R., Martin, N., Robotham, A., Venn, K., Villaver, E., Bovy, J., Boselli, A., Colless, M., Comparat, J., Denny, K., Duc, P.-A., Ellison, S., de Grijs, R., Fernandez-Lorenzo, M., Freeman, K., Guhathakurta, R., Hall, P., Hopkins, A., Hudson, M., Johnson, A., Kaiser, N., Koda, J., Konstantopoulos, I., Koshy, G., Lee, K.-G., Nusser, A., Pancoast, A., Peng, E., Peroux, C., Petitjean, P., Pichon, C., Poggianti, B., Schmid, C., Shastri, P., Shen, Y., Willot, C., Croom, S., Lallement, R., Schimd, C., Smith, D., Walker, M., Willis, J., Colless, A. B. M., Goswami, A., Jarvis, M., Jullo, E., Kneib, J.-P., Konstantopoloulous, I., Newman, J., Richard, J., Sutaria, F., Taylor, E., van Waerbeke, L., Battaglia, G., Hall, P., Haywood, M., Sakari, C., Schmid, C., Seibert, A., Thirupathi, S., Wang, Y., Wang, Y., Babas, F., Bauman, S., Caffau, E., Laychak, M. B., Crampton, D., Devost, D., Flagey, N., Han, Z., Higgs, C., Hill, V., Ho, K., Isani, S., Mignot, S., Murowinski, R., Pandey, G., Salmon, D., Siebert, A., Simons, D., Starkenburg, E., Szeto, K., Tully, B., Vermeulen, T., Withington, K., Arimoto, N., Asplund, M., Aussel, H., Bannister, M., Bhatt, H., Bhargavi, S., Blakeslee, J., Bland-Hawthorn, J., Bullock, J., Burgarella, D., Chang, T.-C., Cole, A., Cooke, J., Cooper, A., Di Matteo, P., Favole, G., Flores, H., Gaensler, B., Garnavich, P., Gilbert, K., GonzalezDelgado, R., Guhathakurta, P., Hasinger, G., Herwig, F., Hwang, N., Jablonka, P., Jarvis, M., Kamath, U., Kewley, L., Le Borgne, D., Lewis, G., Lupton, R., Martell, S., Mateo, M., Mena, O., Nataf, D., Newman, J., Pérez, E., Prada, F., Puech, M., Recio-Blanco, A., Robin, A., Saunders, W., Smith, D., Stalin, C. S., Tao, C., Thanjuvur, K., Tresse, L., van Waerbeke, L., Wang, J.-M., Yong, D., Zhao, G., Boisse, P., Bolton, J., Bonifacio, P., Bouchy, F., Cowie, L., Cunha, K., Deleuil, M., de Mooij, E., Dufour, P., Foucaud, S., Glazebrook, K., Hutchings, J., Kobayashi, C., Kudritzki, R.-P., Li, Y.-S., Lin, L., Lin, Y.-T., Makler, M., Narita, N., Park, C., Ransom, R., Ravindranath, S., Eswar Reddy, B., Sawicki, M., Simard, L., Srianand, R., Storchi-Bergmann, T., Umetsu, K., Wang, T.-G., Woo, J.-H., and Wu, X.-B., "The Detailed Science Case for the Maunakea Spectroscopic Explorer: the Composition and Dynamics of the Faint Universe," ArXiv e-prints (May 2016).

[28] Noll, S., Kausch, W., Barden, M., Jones, A. M., Szyszka, C., Kimeswenger, S., and Vinther, J., "An atmospheric radiation model for Cerro Paranal. I. The optical spectral range," A\&A 543, A92 (July 2012).

[29] Jones, A., Noll, S., Kausch, W., Szyszka, C., and Kimeswenger, S., "An advanced scattered moonlight model for Cerro Paranal," A\&A 560, A91 (Dec. 2013).

[30] Flagey, N., Mignot, S., and Szeto, K., "Modeling and budgeting fiber injection efficiency for the Maunakea spectroscopic explorer (MSE)," in [Modeling, Systems Engineering, and Project Management for Astronomy VIII], Proc. SPIE 10705 (Aug. 2018). 